\title{
Tumor Boards during COVID-19 Pandemic
}

\author{
Ahmed Nadeem Abbasi \\ Department of Oncology, The Aga Khan University, Karachi, Pakistan
}

COVID-19 pandemic is a global healthcare issue. Cancer patients management also got effected due to the emergency measures adopted by the healthcare policy-makers in order to provide urgent services to COVID-19 patients. In the recent few weeks, some guidelines and directives are being published and circulated, which are being followed by cancer-care providers. ${ }^{1,2}$

Globally, the effects of this COVID-19 pandemic were felt by both healthcare providers and healthcare policy-makers. This unprecedented pandemic is being faced across the world in all countries; hence, every country came up with national plans of service provision that addressed not only the COVID-19 patients' care, but also define guidelines for the treatment of cancer patients during this difficult period. ${ }^{3}$ The ultimate beneficiary of modern well-networked cancer-care systems is the patient. Cancer patients care and management is also being considered as a serious task by healthcare policy-makers. As a first step in cancer management, a site-specific multi-disciplinary tumor board is conducted. When it comes to cancer patients treatment, recommendations made at site-specific multidisciplinary tumor boards form the basis of a comprehensive plan of management. ${ }^{4}$

During this pandemic, it is not practically possible to gather colleagues in a conference room for multi-disciplinary tumor board, due to the restrictions as per directives aiming towards the control of this pandemic. These board meetings are open to all colleagues who are providing cancer-care in other hospitals as well. Instead of cancelling these tumor boards, site-specific teams have decided to take the help of information technology expert colleagues and to conduct on-line web-based meetings in which clinicians discuss their cases in the same way as they were doing before the pandemic. The quality of cross-deliberations was found to be similar during these on-line meetings. The concept of multi disciplinary interaction remained intact and concerned doctors were able to involveall relevantspecialty doctors. Active participation of junior trainee postgraduate residents and under-graduate students was also observed. The objective of sharing this experience was to encourage the colleagues, across the world to consider making this arrangement for their respective site-specific tumor boards.

Correspondence to: Dr. Ahmed Nadeem Abbasi, Department of Oncology, The Aga Khan University, Stadium Road, Karachi, Pakistan

E-mail: nadeem135@gmail.com

Received: April 14, 2020; Revised: April 18, 2020;

Accepted: May 04, 2020

DOI: https://doi.org/10.29271/jcpsp.2020.JCPSPCR.CR1
This is a useful take-home message for the specialist colleagues, who are working in the developing countries. As far as our provision of cancer-care processes are concerned, we strongly recommend the establishment of high quality sitespecific tumor boards in every institution where cancer patients are being treated. We consider these tumor boards as a lifeline of cancer patients in developing countries. ${ }^{5}$

An International Collaborative Group has published a recommended practical approach pertaining to our day-to-day clinical services, which we are providing to our cancer patients in this very difficult time of history when the whole world is facing a serious pandemic. ${ }^{6}$ Wearefully aware of thefact that site-specific multidisciplinary tumor board plays a pivotal role in the overall comprehensive cancer-care plan.

We have to discuss our cases before offering the first modality of treatment to patients. This is the only way we can ascertain that best management plan is being recommended to these patients. It is advisable to conduct these tumor board meetings with an open access to both junior and senior doctors of all concerned specialties.

Innovative measures are required for the continuation of all quality practices, processes and procedures during the time of this COVID-19 pandemic, which is a global problem. If we will not be able to conduct our tumor boards during this time of pandemic then eventually the outcome of patients will not be the same as before. Large conference rooms are being used for these meetings. These site-specific boards are an important integral part of students' clinical rotations. In certain circumstances, these multidisciplinary tumor boards can be conducted via web link as we are doing in case of pediatric neuro-oncology tumor board, which is being conducted once a month. This is a video-linked meeting in which few centres of Pakistan get connected with the Sick Kids Hospital, Toronto, Canada for the discussion of Pakistani cancer children cases. During the time of COVID-19 pandemic, the same strategy was followed and now we are conducting majority of our tumor boards via networking softwares, which can be operated on personal mobile phones and home computers as well. In this way, all stakeholders can give their expert input pertaining to their specific specialty. Tumor board discussions have to be continued in the same manner until this pandemic is over; and then we can come back to our conventional way of sitting together in a room for discussion, debate and deliberation on each case presented at the tumor board meeting. 


\section{REFERENCES}

1. De Paula BHR, Araújo I, Bandeira L, Barreto NMPB, Doherty G]. Recommendations from national regulatory agencies for ongoing cancer trials during the COVID-19 pandemic. Lancet Oncol 2020; 21(5):624-7.

2. Ueda M, Martins R, Hendrie PC, McDonnell T, Crews JR, Wong $\mathrm{TL}$, et al. Managing cancer care during the covid-19 pandemic: Agility and collaboration toward a common goal. J Natl Compr Canc Netw 2020; 1-4 [Online ahead of print].

3. Combs SE, Belka C, Niyazi M, Corradini S, Pigorsch S, Wilkens J, et al. First statement on preparation for the COVID-19 pandemic in large German speaking universitybased radiation oncology departments. Radiat Oncol 2020;
15(1):74.

4. Porzio G, Cortellini A, Bruera E, Verna L, Ravoni G, Peris F, et al. Home care for cancer patients during COVID-19 pandemic: The "double triage" protocol. J Pain Symptom Manage 2020; S0885-3924(20)30172-X.

5. Abbasi AN, Karim MU, Ali N, Hafiz A, Qureshi BM. Multidisciplinary team tumour boards are a lifeline for our cancer patients in lower and middle income countries. Clin Oncol (R Coll Radiol) 2016; 28(12):799.

6. Al-Shamsi HO, Alhazzani W, Alhuraiji A, Coomes EA, Chemaly RF, Almuhanna M, et al. A practical approach to the management of cancer patients during the novel coronavirus disease 2019 (covid-19) pandemic: An international collaborative group. Oncologist 2020. doi: 10.1634/ theoncologist.2020-0213 [Online ahead of print]. 